\title{
Bioinspired Bifunctional Membrane for Efficient Clean Water Generation
}

Yang Liu,,$\stackrel{a}{\ddagger}$ Jinwei Lou, ${ }^{a}, \$$ Mengtian Ni, ${ }^{a}$ Chengyi Song, ${ }^{a}$ Jianbo Wu, ${ }^{a}$ Neil P.

Dasgupta, ${ }^{\mathrm{b}}$ Peng Tao, ${ }^{\mathrm{a},}$ " Wen Shang, ${ }^{\mathrm{a},}$ " Tao Deng ${ }^{\mathrm{a}}$

${ }^{a}$ State Key Laboratory of Metal Matrix Composites, School of Materials Science and Engineering, Shanghai Jiao Tong University, 800 Dongchuan Rd, Shanghai, 200240, P. R. China

${ }^{\mathrm{b}}$ Department of Mechanical Engineering, University of Michigan, Ann Arbor, Michigan 48109, United States

$\$$ Yang Liu and Jinwei Lou contributed to this work equally. 


\section{Experimental details}

\section{SEM- energy dispersive spectroscopy (EDS) measurement}

The cross-sectional elemental distribution of the $\mathrm{TiO}_{2} / \mathrm{Au} / \mathrm{AAO}$ 3-layered membranes was collected by an Analytical Field Emission SEM (JEOL JSM-7800F Prime) with EDS (Thermo Scientific TM NORANTM System 7) operated at an acceleration voltage of $5 \mathrm{kV}$ under the mapping mode. The SEM sample was prepared by breaking the $\mathrm{TiO}_{2} / \mathrm{Au} / \mathrm{AAO}$ 3-layered membrane to expose the cross-section.

\section{Bifunctional membranes with thinner $\mathrm{TiO}_{2}$ layer}

To prepare membrane with a thinner $\mathrm{TiO}_{2} \mathrm{NP}$ film layer, aqueous $\mathrm{TiO}_{2} \mathrm{NP}$ dispersion with total mass of $0.2 \mathrm{mg}$ was filtrated through the prepared Au/AAO 2-layered membrane. Commercial $\mathrm{TiO}_{2} \mathrm{NPs}$ were mixed with DI water and the dispersion was thoroughly sonicated. The obtained dispersion was sit for $1 \mathrm{~h}$ for the large aggregates to precipitate naturally due to gravity. Only the top stable dispersion was then used for the preparation of the $\mathrm{TiO}_{2} \mathrm{NP}$ film layer through filtration. The cross-sectional microstructure images of the prepared membrane were collected by SEM (FEI Sirion 200) at both low and high magnifications.

\section{Au/AAO membranes with different Au NP size and mass}

$\mathrm{Au}$ NPs with diameters of both $50 \mathrm{~nm}$ and $100 \mathrm{~nm}$ were synthesized through the same citrate reduction and growth processes. The obtained aqueous Au NP dispersions were filtered through the AAO substrate to prepare 2-layered Au/AAO membranes with a continuous Au NP film covering on top of the AAO substrate. Generally mass of Au NPs less than $0.18 \mathrm{mg}$ leads to the formation of non-continuous films. Water evaporation performance of the prepared membranes was tested under a solar illumination $(5 \mathrm{~W}$ ) for $15 \mathrm{~min}$. The water weight loss was measured with digital balance in real time. 


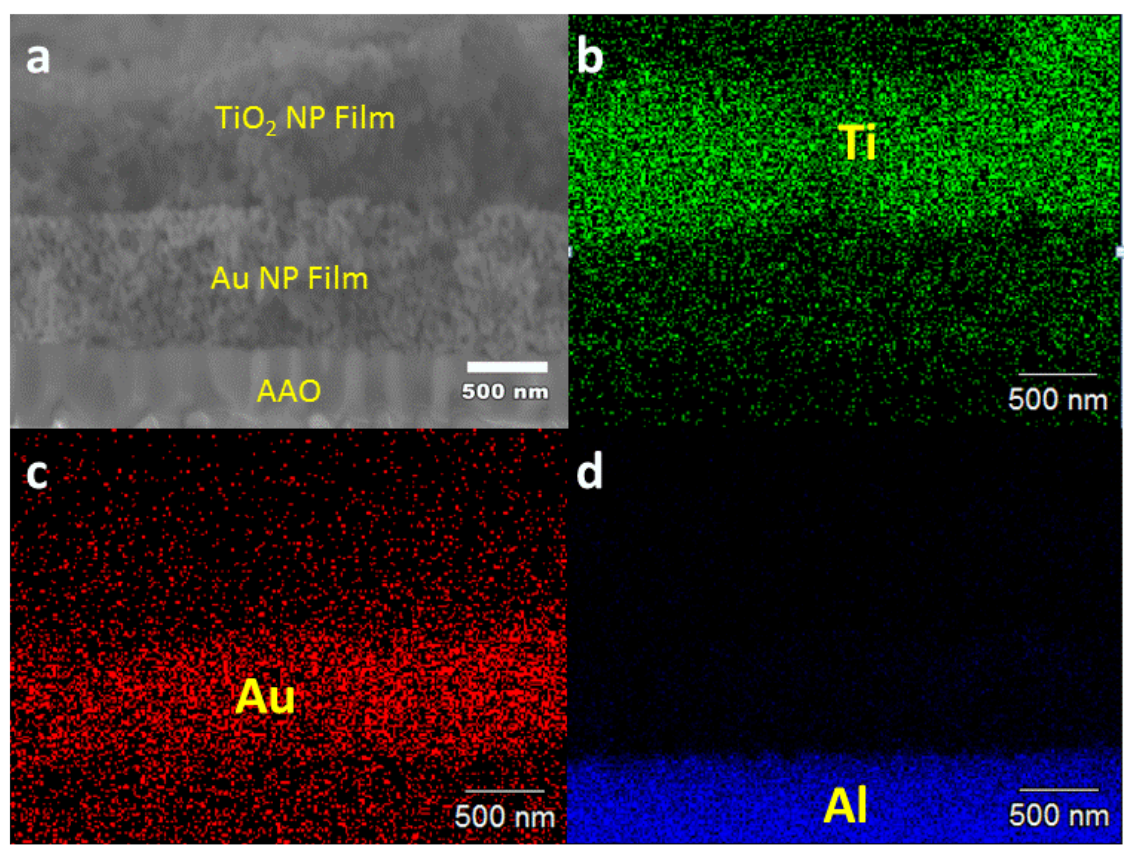

Figure S1. EDS characterization of bifunctional membrane: (a) A cross-sectional SEM image showing the distinct 3-layer structure of $\mathrm{TiO}_{2} \mathrm{NP}$ film on the top, Au NP film in the middle and AAO substrate at the bottom. (b-d) EDS mapping images of Ti, $\mathrm{Au}$, and $\mathrm{Al}$ elemental distribution.
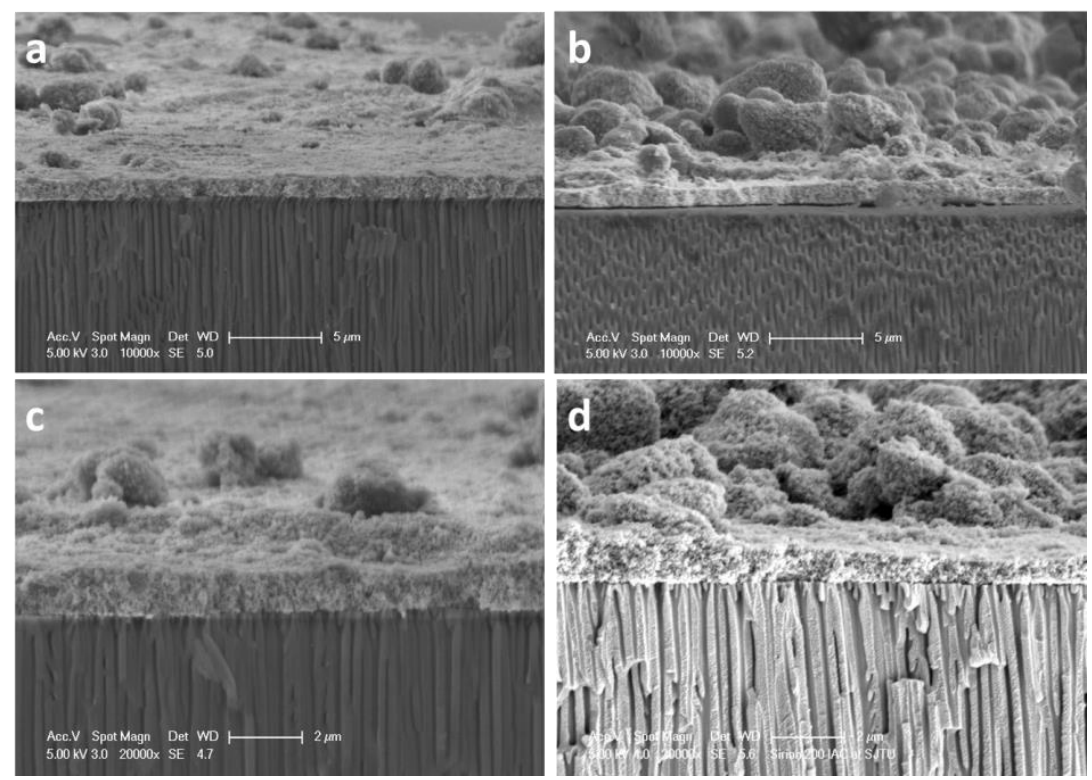

Figure S2. Cross-sectional SEM images of bifunctional membrane with different $\mathrm{TiO}_{2} \mathrm{NP}$ contents: (a, c) $0.2 \mathrm{mg}$ of $\mathrm{TiO}_{2} \mathrm{NPs}$ at low and high resolutions; (b, d) $0.5 \mathrm{mg}$ of $\mathrm{TiO}_{2} \mathrm{NPs}$ at low and high resolutions. 
a

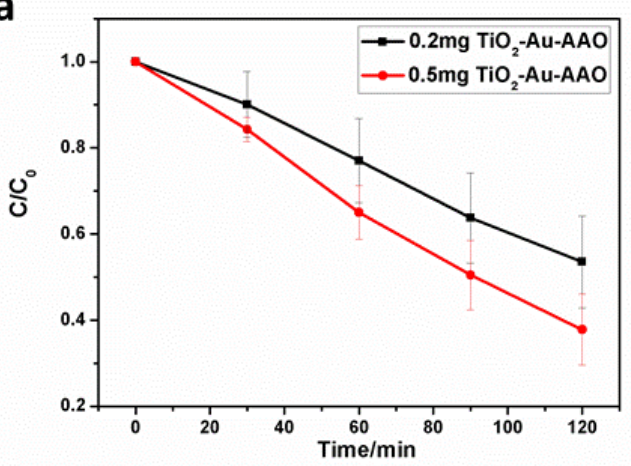

b

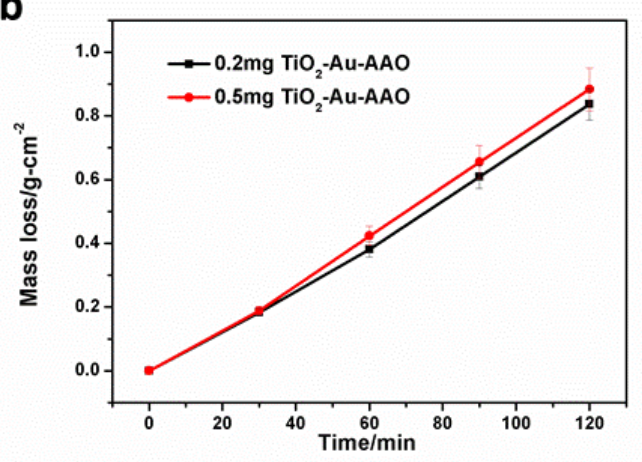

Figure S3. (a) Photocatalytic degradation of RhB using bifunctional membrane with different $\mathrm{TiO}_{2} \mathrm{NP}$ contents; (b) Evaporation performance using bifunctional membrane with different $\mathrm{TiO}_{2}$ NP contents.

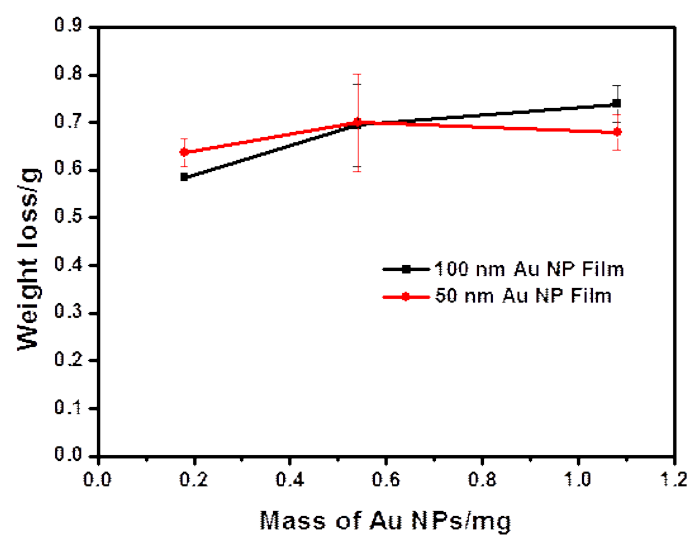

Figure S4. Water evaporation performance of continuous films of Au NP (50 nm and $100 \mathrm{~nm}$ ) with different NP contents. Weight losses were measured after 15 mins of solar light illumination. 